\title{
Comment on Flagel et al.: Sign-tracking as a predictor of addiction vulnerability
}

\author{
Veronika Pohořalá ${ }^{1} \cdot$ Thomas Enkel $^{2} \cdot$ Dusan Bartsch $^{2} \cdot$ Rainer Spanagel $^{1} \cdot$ Rick E. Bernardi $^{1}$
}

Received: 14 June 2021 / Accepted: 6 July 2021 / Published online: 29 July 2021

(c) The Author(s) 2021

Flagel et al., in their Letter to the Editor Sign-tracking as a predictor of addiction vulnerability, present a reasoned and valuable comment on our recent study (Pohorala et al. 2021) that outlines the seminal work on sign- and goal-tracking and how these behaviors contribute to drug cue-mediated responding in animals. We concur with most of what the authors have written; however, we do not entirely agree with their seeming conclusion that only a defined set of experimental measurements should be used to uncover cognitive-motivational traits that may underlie phenotypes associated with sign- and goaltracking. Although not all of the conditions of our study-as Flagel et al. rightfully point out in their letter-were those with which behavioral differences between sign-tracking and goal-tracking have typically been most apparent, it was in fact the objective of our study to specifically evaluate whether a specific phenotype (sign-tracking) demonstrated under a given set of conditions (discrete drug-associated cues) exhibited similar addiction-like behaviors under a different set of experimental conditions (the 3-CRIT protocol). Similarly, it must be emphasized that the value of the sign-tracking phenotype with respect to addiction lies-again as the authors state-in its ability to predict addiction vulnerability. And because the 3-CRIT model is, to date, arguably the most translationally relevant multisymptomatic model of addiction available, it stands to reason that phenotypes associated with sign-tracking

Veronika Pohořalá and Thomas Enkel share the 1st authorship.

Rick E. Bernardi

rick.bernardi@zi-mannheim.de

1 Institute of Psychopharmacology, Central Institute of Mental Health, Medical Faculty Mannheim, University of Heidelberg, J5 68159 Mannheim, Germany

2 Department of Molecular Biology, Central Institute of Mental Health, Medical Faculty Mannheim, University of Heidelberg, Mannheim, Germany might, in fact, relate to and predict addictive-like behavior in the 3-CRIT model. In other words, we thought it logical that enhanced cue salience associated with the sign-tracking phenotype might indeed manifest itself in other drug-seeking behaviors, including those measured using the 3-CRIT model. This was hypothesized to be especially relevant considering that 3-CRIT animals have previously demonstrated increased cue and cocaine-induced reinstatement relative to 0-CRIT animals (Cannella et al. 2017, 2013), as well as increased cue-mediated cocaine-seeking following a withdrawal period (unpublished results). These findings are of course consistent with those previously demonstrated in sign-trackers relative to goal-trackers (Saunders and Robinson 2011). Unfortunately, a marriage of these two models, in this experiment under these conditions, examining along the range of Pavlovian approach scores among sign-trackers, intermediates, and goal-trackers, did not result in a correlation with 3-CRIT measures. However, we continue to believe that, given the relevance of the sign-tracking phenotype to vulnerability to addiction-like characteristics in animal models despite limited translational validity, as well as the critical information provided by the 3-CRIT model over the last several years, there may still be a way to bridge these two paradigms. Nonetheless, we are grateful to Flagel et al. for their Letter to the Editor, which provides some welcome further context and nuance to our findings.

Funding Open Access funding enabled and organized by Projekt DEAL. Financial support for this work was provided by the Bundesministerium für Bildung und Forschung (BMBF)-funded SysMedSUDs consortium (FKZ: 01ZX1909A) and the Deutsche Forschungsgemeinschaft (DFG, German Research Foundation) - Project-ID 402170461 - TRR 265.

\section{Declarations}

Competing interests The authors declare no competing interests. 
Open Access This article is licensed under a Creative Commons Attribution 4.0 International License, which permits use, sharing, adaptation, distribution and reproduction in any medium or format, as long as you give appropriate credit to the original author(s) and the source, provide a link to the Creative Commons licence, and indicate if changes were made. The images or other third party material in this article are included in the article's Creative Commons licence, unless indicated otherwise in a credit line to the material. If material is not included in the article's Creative Commons licence and your intended use is not permitted by statutory regulation or exceeds the permitted use, you will need to obtain permission directly from the copyright holder. To view a copy of this licence, visit http://creativecommons.org/licenses/by/4.0/.

\section{References}

Cannella N, Cosa-Linan A, Roscher M, Takahashi TT, Vogler N, Wängler B et al (2017) [18F]-Fluorodeoxyglucose-positron emission tomography in rats with prolonged cocaine self-administration suggests potential brain biomarkers for addictive behavior. Front Psych 8:1-14
Cannella N, Halbout B, Uhrig S, Evrard L, Corsi M, Corti C et al (2013) The mGluR2/3 agonist LY379268 induced anti-reinstatement effects in rats exhibiting addiction-like behavior. Neuropsychopharmacol 38:2048-2056

Pohorala V, Enkel T, Bartsch D, Spanagel R, Bernardi RE (2021) Sign- and goal-tracking score does not correlate with addictionlike behavior following prolonged cocaine self-administration. Psychopharmacology (Berl) 238:2335-2346

Saunders BT, Robinson TE (2011) Individual variation in the motivational properties of cocaine. Neuropsychopharmacol $36: 1668-1676$

Publisher's note Springer Nature remains neutral with regard to jurisdictional claims in published maps and institutional affiliations. 